\title{
CaRotid Artery Filtering Technique (CRAFT): A Technique for Carotid Artery Stenting with Intrinsic Embolic Protection
}

\author{
Michelle Foo, $\mathrm{MBBS}^{1}$, Yifan Ren, $\mathrm{MD}^{1}$, Jay Gajera, $\mathrm{MD}^{2}$, Christen D Barras, FRANZCR ${ }^{3,4}$, \\ Hong Kuan Kok, FRANZCR ${ }^{5,6}$, Ashu Jhamb, FRANCR7, Hussein Abbouchie, MD ${ }^{1}$, \\ Julian Maingard, FRANZCR ${ }^{6,8}$, Duncan Mark Brooks, FRANZCR ${ }^{1,9}$, Hamed Asadi, FRANZCR $^{1,6,7,8,9}$ \\ ${ }^{1}$ Department of Radiology, Austin Hospital, Melbourne, Vic, Australia \\ ${ }^{2}$ Department of Radiology, Alfred Health, Melbourne, Vic, Australia \\ ${ }^{3}$ South Australian Health and Medical Research Institute, University of Adelaide, Adelaide, SA, Australia \\ ${ }^{4}$ Department of Radiology, Royal Adelaide Hospital, Adelaide, SA, Australia \\ ${ }^{5}$ Interventional Radiology Service, Department of Radiology, Northern Health, Melbourne, Vic, Australia \\ ${ }^{6}$ School of Medicine, Faculty of Health, Deakin University, Waurn Ponds, Vic, Australia \\ ${ }^{7}$ Interventional Radiology Service, Department of Radiology, St Vincent's Hospital, Melbourne, Vic, Australia \\ ${ }^{8}$ Interventional Neuroradiology Service, Department of Radiology, Monash Health, Melbourne, Vic, Australia \\ ${ }^{9}$ Stroke Theme, The Florey Institute of Neuroscience and Mental Health, University of Melbourne, Melbourne, Vic, Australia
}

Purpose: Carotid artery stenting (CAS) is an established treatment for symptomatic carotid artery stenosis as an alternative to carotid endarterectomy. A variety of techniques and devices have been devised to minimise periprocedural stroke risk using either proximal or distal embolic protection. This study presents a method of embolic protection during CAS-the CaRotid Artery Filtering Technique (CRAFT).

Materials and Methods: The CRAFT technique employs aspects of both proximal and distal embolic protection. The CASPER RX stent (MicroVention, Tustin, CA, USA), which is a double-layered, closed-cell, micromesh nitinol stent, is deployed across the carotid artery stenosis with the assistance of a FlowGate balloon guide catheter (Stryker Neurovascular, Fremont, CA, USA). The partially deployed stent acts as a distal filter while the balloon guide is deflated midway during stent deployment to prevent distal plaque embolisation, followed by completion of stent deployment and angioplasty.

Results: A total of 94 patients underwent CAS using the CRAFT technique between June 2016 and March 2021. Successful stent deployment was achieved in all patients. Preliminary results demonstrated acute stent occlusion in 6 patients (6.4\%) and distal embolic stroke in 5 patients (5.3\%). The median procedural fluoroscopy time was 34 minutes with an interquartile range of 22 to 55 minutes.

Conclusion: The CRAFT technique of CAS presented by this study can be applied in the treatment of symptomatic carotid artery stenosis in both emergency and elective procedure settings with a high technical success and low distal embolic stroke risk.

Key Words: Carotid arteries; Stents; Radiology; Interventional

\section{Correspondence to: Yifan Ren, MD \\ Department of Radiology, Austin Hospital, 145 Studley Rd, Heidelberg, Melbourne, Vic 3084, Australia \\ Tel: +61422653000 \\ Fax: +61394964779 \\ E-mail: rendaxing0@gmail.com}

Received: July 12, 2021

Revised: October 5, 2021

Accepted: October 6, 2021

Copyright $\odot 2021$ Korean Society of Interventional Neuroradiology This is an Open Access article distributed under the terms of the Creative Commons Attribution Non-Commercial License (http://creativecommons.org/licenses/by-nc/4.0) which permits unrestricted non-commercial use, distribution, and reproduction in any medium, provided the original work is properly cited. 


\section{INTRODUCTION}

Carotid artery stenting (CAS) has an established role in the management of symptomatic carotid artery stenosis. Advancements in imaging, endovascular devices, and techniques have enabled improved technical and clinical outcomes. Whilst CAS offers many benefits over carotid endarterectomy (CEA), such as avoidance of general anaesthesia, injury to adjacent neurovascular structures, reduced risk of wound infection, and reduced length-of-stay, concerns still exist regarding the risk of periprocedural stroke-the most common major complication of CAS. ${ }^{1}$ However, large scale clinical trials have demonstrated that the long-term functional outcome, risk of disabling stroke, myocardial infarction or death did not differ significantly between CAS and CEA. ${ }^{2,3}$ In addition, numerous devices and technical methods, including new stents and embolic protection devices (EPD), have been designed and trialled, aiming to further reduce the risk of cerebral embolisation during CAS. However, there is still a lack of clear consensus on which combination of strategies is most efficacious.

As a result, substantial heterogeneity exists in the current practice of CAS across different centres and operators, and innovation is needed to establish an ideal technique. The present study describes the technique, efficacy and safety of a method of CAS utilising aspects of both distal and proximal embolic protection, entitled "CaRotid Artery Filtering
Technique" (CRAFT)

\section{MATERIALS AND METHODS}

This is a retrospective study performed in two comprehensive stroke centres in Australia to evaluate the technical success and peri-procedure complication of CAS using CRAFT. All patients who underwent CAS for symptomatic carotid artery stenosis or occlusion using the CASPER RX stent with CRAFT technique between June 2016 and March 2021 were included in the study. All emergency patients received clinical assessment, computed tomography (CT) angiogram and perfusion studies which confirmed their eligibility for carotid stenting and/or endovascular clot retrieval. All CAS procedures were performed in biplane angiography suites by experienced interventional neuroradiologists. Procedural success and peri-procedural complications were collected from electronic clinical records. This study was approved by the institutional human research ethics committee.

\section{Device Description}

The CASPER RX stent (Roadsaver in Europe), "Carotid Artery Stent designed to Prevent Embolic Release" (MicroVention, Tustin, (A, USA), is a nitinol, self-expanding, double-layered micromesh stent with flared ends. The stent has a closed-cell design with tiny cells $(375-700 \mu \mathrm{m})$ and is available in various
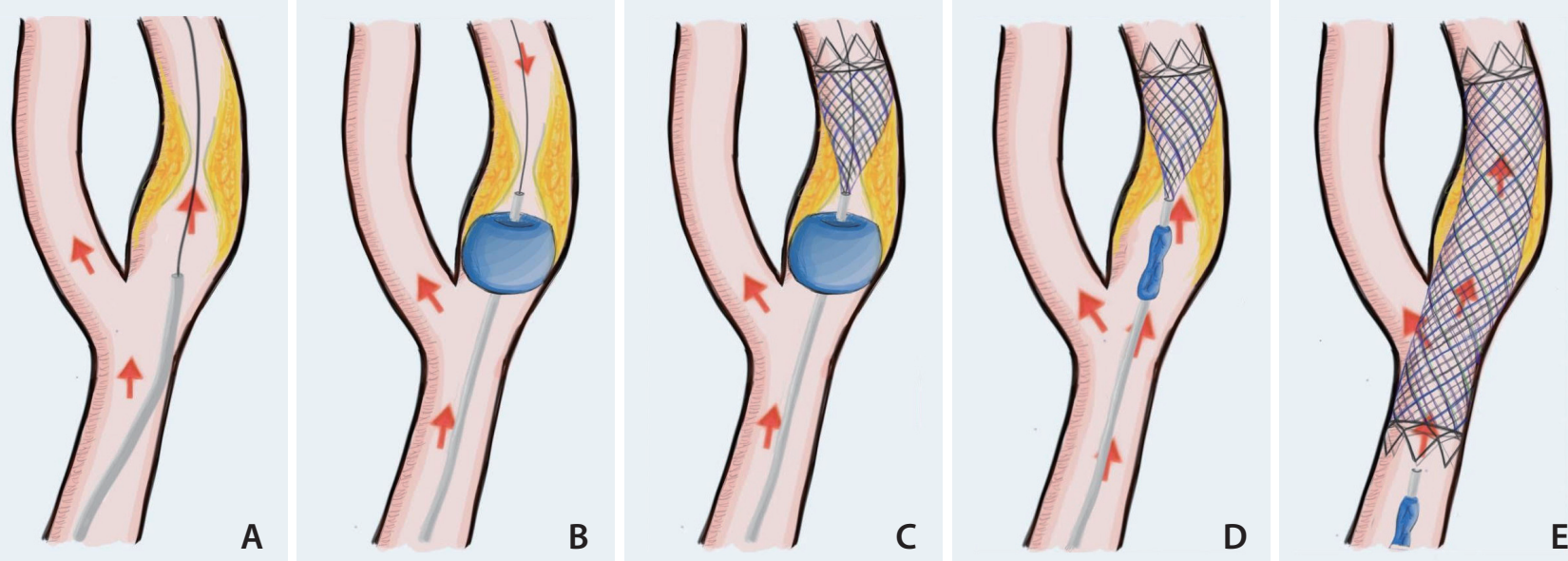

Fig. 1. Diagrammatic stepwise breakdown of deploying CASPER RX stent (MicroVention, Tustin, CA, USA) with the assistance of balloon across carotid artery stenosis (CaRotid Artery Filtering Technique, CRAFT). (A) FlowGate Balloon Guide Catheter (BGC) (Stryker Neurovascular, Kalamazoo, MI, USA) and its supplied inner catheter are advanced to the internal carotid artery (ICA), just proximal to the lesion and lesion crossed by guidewire. (B) BGC inflated in ICA causing an arrest of antegrade flow. (C) A CASPER RX stent introduced over the guidewire into the ICA, distal to the lesion and partially deployed. (D) BGC is deflated to restore antegrade flow. (E) Stent is completely deployed into the ICA and CCA. Red arrow indicates direction of blood flow. 
sizes, with outer diameters ranging from 5-10 $\mathrm{mm}$ and double-layer length ranging from $18-40 \mathrm{~mm}$. The struts have a braided design, allowing conformation to the tortuosity within the internal carotid artery (ICA) and common carotid artery (CCA). The delivery system comprises a 5-French $143 \mathrm{~cm}$ length rapid exchange delivery catheter over a 0.014 inch guidewire platform.

\section{CRAFT Technique}

An 8-French introducer sheath is inserted into the right common femoral artery.

In cases of isolated ICA occlusion, an 8-French FlowGate Balloon Guide Catheter (BGC) (Stryker Neurovascular, Kalamazoo, MI, USA) and its supplied inner catheter are advanced to the CCA or ICA, just proximal to the lesion. An 0.014 inch $\times 200$ or $300 \mathrm{~cm}$ guidewire (Synchro'; Stryker Neurovascular) (Fig. 1A) is then used to cross the ICA lesion. The BGC is inflated in the CCA or ICA, causing an arrest of antegrade flow (Fig. 1B). A CASPER RX stent (MicroVention) is introduced over the guidewire into the ICA, distal to the lesion. Prior to loading on to the guidewire, the CASPER stent is flushed with anti-IIb/IIla inhibitor, with the intention to reduce the risk of stent occlusion. The CASPER RX stent is then partially deployed (distal third) such that the double-layered segment of the stent is well apposed to the distal vessel wall (Fig. 1C). At this point, the BGC is deflated to restore antegrade flow, and the partially deployed distal stent acts as an embolic filter due to the micromesh nature of the stent (Fig. 1D). Finally, the stent is completely deployed into the CCA, and angioplasty performed with a 5 or $5.5 \mathrm{~mm}$ non-compliant monorail coronary angioplasty balloon (Sterling; Boston Scientific, Marlborough, MA, USA) (Fig. 1E).

In cases of a tandem occlusion, an 8-French FlowGate BGC is inserted over a 0.035 inch guidewire (GlideWire; Terumo, Tokyo, Japan) into the CCA, where angiography is performed to confirm the ICA occlusion. The FlowGate balloon is inflated as the ICA occlusion is crossed using a coaxial system, comprising a 5-French intermediate catheter (SOFIA; MicroVention); a 0.014 inch microwire (Synchro ${ }^{2}$ ) and either a Trevo Pro 18 (Stryker Neurovascular) or Headway 21 microcatheter (MicroVention). The microcatheter is navigated past the intracranial occlusion over the microwire, and stent retriever thrombectomy is performed with either a Solitaire (Medtronic, Irvine, CA, USA) or Trevo (Stryker Neurovascular) device of appropriate size. Vacuum syringe suction is applied to the intermediate catheter, through which the stent retriever de- vice is removed. After satisfactory revascularisation, a 0.014 inch $\times 200$ or $300 \mathrm{~cm}$ length microwire is introduced via the intermediate catheter, in preparation for CAS. The intermediate catheter is then removed before introducing the CASPER RX stent over the microwire into the cervical ICA, beyond the occlusion. As previously described, the FlowGate balloon guide, still proximal to the occlusion, is inflated before partial stent deployment and the distal third of the stent is used as a filter upon deflating the balloon. Complete stent deployment and angioplasty ensue.

If difficulty is encountered upon navigating the microcatheter to the intracranial occlusion, the BGC may be advanced intracranially, through the ICA occlusion, to offer greater distal stability. After stent retriever thrombectomy, the BGC may then be withdrawn to the cervical ICA, just distal to the occlusion, where it is inflated for partial stent deployment, then deflated for stent filtration and full stent deployment.

\section{Antiplatelet Therapy}

Our experience led us to apply following antiplatelet therapy for patients who received CASPER RX stent with CRAFT technique. In addition, all patients included in this study received antiplatelet therapy as below regardless of whether they received intravenous tissue plasminogen activator or not.

For emergency cases with acute presentations, all patients received 500 mg intravenous aspirin followed by 2,000-3,000 units of intravenous heparin when the balloon was inflated. Depending on the time of symptoms onset and the amount of initial ischemic core demonstrated on CT perfusion, repeat CT brain study was performed $2-4$ hours after the procedure to confirm the size of ischaemic core and to assess for intracranial haemorrhage. Based the result, the patient received an additional single dose of either 600 or 300 mg clopidogrel.

For elective cases, depending on the acuity of presentation, all patients received a loading dose of $600 \mathrm{mg}$ aspirin and $600 \mathrm{mg}$ clopidogrel 3-5 days prior to the procedure followed by a daily dose of $100 \mathrm{mg}$ aspirin and $75 \mathrm{mg}$ of clopidogrel including the day of procedure. During the procedure, prior to stent deployment, $500 \mathrm{mg}$ intravenous aspirin was administered if there was any concern regarding a patient's platelet aggregation. In addition, 2,000 units of intravenous heparin was administered when the balloon was inflated.

Following CAS, when possible, all patients were commenced on dual antiplatelet therapy consisting of $75 \mathrm{mg}$ 
clopidogrel daily and $100 \mathrm{mg}$ aspirin daily for 6-12 months followed by daily aspirin for several years depend on the clinical status.

\section{RESULTS}

A total of 89 patients underwent 94 cases of CAS using the CRAFT technique between June 2016 and March 2021. Patient demographics, basic lesion characteristics and mechanical thrombectomy data are summarized in Tables 1 and 2. The effectiveness and technical feasibility of this technique have been described in our previous publication. ${ }^{4}$ In the setting of acute stroke, it was shown that this technique had a high technical success rate (100\%) and a relatively low complication rate.

Successful stent deployment was achieved in all cases. Preliminary results demonstrated acute stent occlusion in 6 cases (6.4\%), and 1 case had recurrent stent occlusion and subsequently died due to extensive middle cerebral artery territory infarct. Five cases (5.3\%) had intra-operative or early peri-operative distal embolic stroke in the middle cerebral artery (MCA) territory, and one of these patients had an ICA tear during the procedure in the setting of angioplasty which led to distal occlusion. All five patients who developed distal embolic stroke had clinical symptoms, and occlusions were confirmed on CT angiogram or digital subtraction angiogram. Patients who had distal occlusions underwent

Table 1. Patient demographics and risk factor profile

\begin{tabular}{lc}
\hline Variable & Value \\
\hline Age (y) & $69 \pm 13$ \\
Sex & \\
Male & $65(73.0)$ \\
Female & $24(27.0)$ \\
Hypertension & $62(69.7)$ \\
Hypercholesterolaemia & $40(44.9)$ \\
Diabetes & $24(27.0)$ \\
Smoker/ex-smoker & $43(48.3)$ \\
Ischaemic heart disease & $19(21.3)$ \\
Previous stroke/TIA & $14(15.7)$ \\
Previous AF & $7(7.9)$ \\
New AF & $9(10.1)$ \\
\hline
\end{tabular}

Values are presented as median \pm standard deviation or number (\%). TIA, transient ischemic attack; AF, atrial fibrillation. successful clot retrieval during the same procedure. Six cases (6.4\%) developed intracranial haemorrhage after the procedure and five of them had symptoms related to the haemorrhage. All of these cases were performed in an emergency setting and three patients were previously on anti-platelet therapy. No other immediate procedure-related complications occurred. The median fluoroscopy time was 34 minutes with an interquartile range of 22 to 55 minutes.

\section{DISCUSSION}

In this study, we describe a CAS technique that employs aspects of both proximal and distal embolic protection, using existing hardware, with excellent technical success and few complications.

Among various modifications in carotid stent design, closed-cell stents, as opposed to open-cell, have the greatest evidence for reducing periprocedural stroke during CAS. In large-scale randomised controlled trials (RCT), closed-cell stents achieved significantly lower rates of major adverse cardiac and cerebrovascular events (stroke, myocardial infarction, death) than open-cell stents within 30 days of CAS. ${ }^{5,6}$ In the last five years, novel double-layered micromesh stents have been used to address concerns regarding plaque protrusion and embolic release through large cells. One recent RCT of 104 patients showed that double-layered stents,

Table 2. Lesion characteristics and mechanical thrombectomy data

\begin{tabular}{lc}
\hline Variable & Value \\
\hline Nature of procedure & \\
Emergency & $86(91.5)$ \\
Elective & $8(8.5)$ \\
Pre-procedure anti-platelet therapy & $30(31.9)$ \\
Emergency & $22(23.4)$ \\
Elective & $8(8.5)$ \\
Carotid lesion side & \\
Right & $49(52.1)$ \\
Left & $45(47.9)$ \\
Tandem lesions on DSA & $57(60.6)$ \\
Location of retrieved tandem lesions & \\
ICA and terminus & $2(5.9)$ \\
ICA and MCA & $55(58.5)$ \\
ICA and ACA & $7(7.4)$ \\
\hline
\end{tabular}

Values are presented as number (\%).

DSA, digital subtraction angiogram; ICA, internal carotid artery; MCA, middle cerebral artery; ACA, anterior cerebral artery. 
compared to single-layered stents, are associated with fewer periprocedural microembolic signals on transcranial Doppler (TCD). However, the overall benefit remains unclear as double-layered stents have also demonstrated a higher risk of acute stent thrombosis. ${ }^{7.8}$

EPD are now routinely used during CAS in the context of early CEA versus CAS trials, such as CREST, showing comparable outcomes when EPD was mandated in CAS. ${ }^{9}$ In 2009, two meta-analyses of trials conducted between 1990 and 2008 also found that EPD were associated with a significant reduction in 30-day stroke or death. ${ }^{10,11}$ However, a subsequent Cochrane meta-analysis, including data from only major RCTS (EVA-3S, SPACE, ICSS), found that EPDs had no significant effect on 30-day stroke or death (odds ratio 0.95 [95\% confidence interval: 0.38-2.41]). ${ }^{2}$ Thus, the exact benefit of EPDs remains unclear, awaiting further high-quality studies, but is broadly recommended (Level B) by the European Society of Vascular Surgery. ${ }^{12}$

Based on our experience, the distal EPD usually has a larger profile than the CASPER stent. In addition, extra steps are required to deploy the EPD distal to the carotid stenosis or occlusion compared to CRAFTS which uses a pre-existing step during carotid stenting. Deploying an EPD distal to the stent can be technically challenging and time-consuming, often
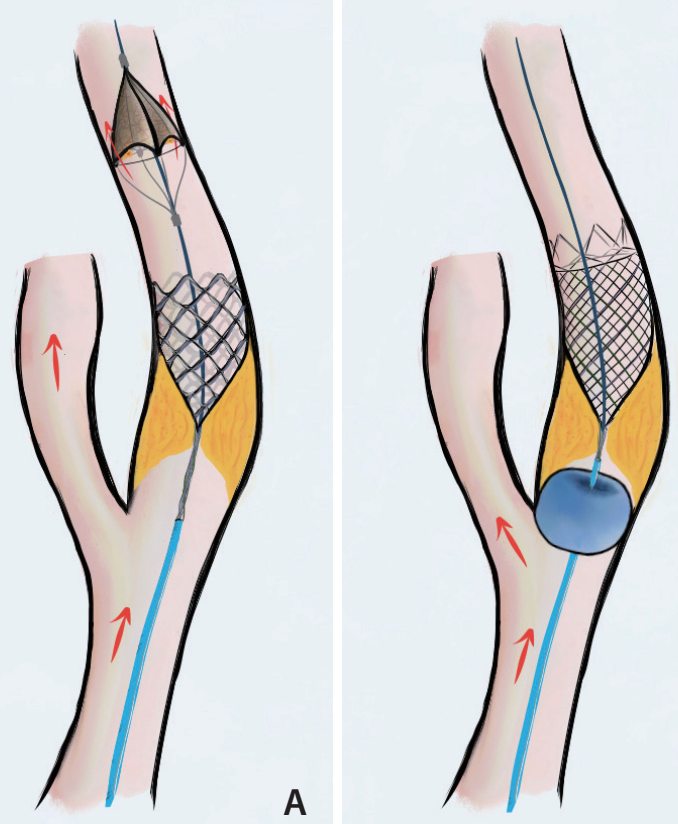

B

Fig. 2. (A) EPD does not fully appose the vessel wall leading to embolus bypassing EPD. (B) Stent fully appose vessel wall. Red arrow indicates direction of blood flow. EPD, embolic protection devices; CRAFT, CaRotid Artery Filtering Technique. under time critical operative conditions. Furthermore, the EPD acts as a distal filter that usually does not fully appose the wall, which can lead to embolus bypassing the EPD and causing distal occlusion when combined with a single layer stent (Fig. 2A). In contrast, the stent is designed to fully appose the wall, providing better coverage with more embolic protection (Fig. 2B).

In particular, speculation exists regarding the type of EPD used: proximal (P-EPD) or distal (D-EPD). P-EPD are occlusion balloons placed in the CCA and external carotid artery (ECA), preventing embolisation by causing an arrest or reverse in blood flow while crossing the ICA stenosis and deploying the stent. D-EPD must cross the stenosis to reach the distal ICA, and act to filter antegrade blood and trap emboli dislodged during the procedure. P-EPDs have proven superior to D-EPD when assessed on the basis of surrogate markers of periprocedural stroke, producing significantly fewer microemboli on diffusion weighted MRI and TCD.8,13,14 Nonetheless, no significant difference has been identified in postoperative neurocognitive decline, stroke, or death between P-EPD and D-EPD, and P-EPD are not without their disadvantages. For example, up to one-third of patients may be neurologically intolerant to P-EPD, manifesting symptoms of ipsilateral cerebral hypoperfusion. 13,15,16 In addition, P-EPD are larger profile devices than D-EPD and may cause vessel wall injury when deployed in the CCA or ECA. As such, P-EPD are contraindicated for patients with contralateral ICA occlusion and patients with advanced CCA or ECA atherosclerosis. ${ }^{12}$

There are several theoretical advantages of the CRAFT approach during CAS. CRAFT achieves distal protection with only the CASPER RX stent, avoiding the need to pass and deploy a separate D-EPD across the occlusion. This removes the extra risk of generating emboli as the D-EPD is passed, and avoids the risk of endothelial injury that occurs when the D-EPD is deployed. Having distal protection in addition to arresting antegrade flow is important because balloon deflation causes a rapid return of blood flow, which exerts shear stress on the carotid plaque, potentially leading to microembolisation of particulate debris. By deflating the balloon during stent deployment rather than afterwards, the duration of cerebral circulatory stasis is reduced, which in itself may cause intracerebral thromboembolism and neurological sequelae. ${ }^{16}$ Although the incidence of distal embolization related to carotid artery stenting in acute ischaemic stroke is not well established, the percentage of cases developing distal embolization in our study (5.3\%) was lower than the 
recent study published by Jadhav et al. ${ }^{17}$ The strength of this study also includes a relatively large cohort size and long study duration. In addition, the procedures were performed by operators with consistent technique.

There are several limitations to this technique. For example, some microemboli may still pass intracranially through gaps between the vessel wall and the single-layered flared end of the CASPER RX stent, especially if not deployed sufficientIy. Moreover, macroemboli may disintegrate upon being captured by the CASPER stent, potentially leading to microemboli travelling through the stent's microcells. In addition, there are also limitations of this study including its retrospective nature across two centres, despite homogeneous CAS practice across all operators. Finally, this study focuses on early periprocedural complications and long-term follow-up data will be the subject of future studies.

\section{CONCLUSION}

This study presents a method of CAS that can be applied in the treatment of symptomatic carotid artery stenosis in both emergent and elective procedure settings. This technique had a high technical success rate but still had a certain periprocedural complication risk. We aim to further establish the validity of this technique by expanding data collection including long-term outcomes.

\section{Fund}

None.

\section{Ethics Statement}

This study was approved by the institutional human research ethics committee. As specific patient information such as age or sex is not included, Informed consent for publication is not required.

\section{Conflicts of Interest}

The authors have no conflicts to disclose.

\section{Author Contributions}

Concept and design: MF, YR, JG, JM, DMB, and HA. Analysis and interpretation: MF, YR, and HA. Data collection: MF, YR, and HA. Writing the article: MF and YR. Critical revision of the article: $C D B, H K K, A J, J M, D M B$, and HA. Final approval of the article: HA. Overall responsibility: MF and YR.

\section{ORCID}

Michelle Foo: https://orcid.org/0000-0001-8056-1163

Yifan Ren: https://orcid.org/0000-0001-6518-6828

Jay Gajera: https://orcid.org/0000-0003-2395-4520

Christen D Barras: https://orcid.org/0000-0003-1899-1909

Hong Kuan Kok: https://orcid.org/0000-0002-8740-6067

Ashu Jhamb: https://orcid.org/0000-0002-7097-1641

Hussein Abbouchie: https://orcid.org/0000-0002-7913-8584

Julian Maingard: https://orcid.org/0000-0001-8958-2411

Duncan Mark Brooks: https://orcid.org/0000-0001-5034-570X

Hamed Asadi: https://orcid.org/0000-0003-2475-9727

\section{REFERENCES}

1. Müller MD, Lyrer P, Brown MM, Bonati LH. Carotid artery stenting versus endarterectomy for treatment of carotid artery stenosis. Cochrane Database Syst Rev 2020;2:CD000515

2. Bonati LH, Gregson J, Dobson J, McCabe DJH, Nederkoorn PJ, van der Worp HB, et al. Restenosis and risk of stroke after stenting or endarterectomy for symptomatic carotid stenosis in the International Carotid Stenting Study (ICSS): secondary analysis of a randomised trial. Lancet Neurol 2018;17:587-596

3. Bonati LH, Dobson J, Featherstone RL, Ederle J, van der Worp $H B$, de Borst GJ, et al. Long-term outcomes after stenting versus endarterectomy for treatment of symptomatic carotid stenosis: the International Carotid Stenting Study (ICSS) randomised trial. Lancet 2015;385:529-538

4. Lamanna A, Maingard J, Kok HK, Barras C, Jhamb A, Thijs V, et al. Carotid artery stenting in acute stroke using a microporous stent device: a single-center experience. World Neurosurg 2019;127:e1003-e1012

5. Doig D, Turner EL, Dobson J, Featherstone RL, Lo RT, Gaines PA, et al. Predictors of stroke, myocardial infarction or death within 30 days of carotid artery stenting: results from the International Carotid Stenting Study. Eur J Vasc Endovasc Surg 2016;51:327334

6. Jansen $\mathrm{O}$, Fiehler J, Hartmann M, Brückmann H. Protection or nonprotection in carotid stent angioplasty: the influence of interventional techniques on outcome data from the SPACE Trial. Stroke 2009:40:841-846

7. Yilmaz $U$, Körner $H$, Mühl-Benninghaus $R$, Simgen A, Kraus $C$, Walter $S$, et al. Acute occlusions of dual-layer carotid stents after endovascular emergency treatment of tandem lesions. Stroke 2017:48:2171-2175

8. Montorsi P, Caputi L, Galli S, Ravagnani PM, Teruzzi G, Annoni A, 
et al. Carotid wallstent versus roadsaver stent and distal versus proximal protection on cerebral microembolization during carotid artery stenting. JACC Cardiovasc Interv 2020;13:403-414

9. Brott TG, Howard G, Roubin GS, Meschia JF, Mackey A, Brooks W, et al. Long-term results of stenting versus endarterectomy for carotid-artery stenosis. N Engl J Med 2016;374:1021-1031

10. Garg N, Karagiorgos N, Pisimisis GT, Sohal DP, Longo GM, Johanning JM, et al. Cerebral protection devices reduce periprocedural strokes during carotid angioplasty and stenting: a systematic review of the current literature. J Endovasc Ther 2009;16:412-427

11. Touzé E, Trinquart L, Chatellier G, Mas JL. Systematic review of the perioperative risks of stroke or death after carotid angioplasty and stenting. Stroke 2009;40:e683-e693

12. Naylor AR. Endarterectomy versus stenting for stroke prevention. Stroke Vasc Neurol 2018;3:101-106

13. Akkaya E, Vuruskan E, Gul ZB, Yildirim A, Pusuroglu H, Surgit O, et al. Cerebral microemboli and neurocognitive change after carotid artery stenting with different embolic protection devices. Int J Cardio/ 2014;176:478-483
14. Bijuklic K, Wandler A, Hazizi F, Schofer J. The PROFI study (Prevention of Cerebral Embolization by Proximal Balloon Occlusion Compared to Filter Protection During Carotid Artery Stenting): a prospective randomized trial. J Am Coll Cardiol 2012;59:13831389

15. Texakalidis P, Letsos A, Kokkinidis DG, Schizas D, Karaolanis G, Giannopoulos S, et al. Proximal embolic protection versus distal filter protection versus combined protection in carotid artery stenting: a systematic review and meta-analysis. Cardiovasc Revasc Med 2018;19(5 Pt A):545-552

16. Giugliano G, Stabile E, Biamino G, Petroni G, Sannino A, Brevetti $\mathrm{L}$, et al. Predictors of carotid occlusion intolerance during proximal protected carotid artery stenting. JACC Cardiovasc Interv 2014;7:1237-1244

17. Jadhav A, Panczykowski D, Jumaa M, Aghaebrahim A, Ranginani $M$, Nguyen F, et al. Angioplasty and stenting for symptomatic extracranial non-tandem internal carotid artery occlusion. J Neurointerv Surg 2018;10:1155-1160 